\title{
Commensurated subgroups, semistability and simple connectivity at infinity
}

\author{
GREGORY R CONNER \\ MichaEL L MiHALIK
}

\begin{abstract}
A subgroup $Q$ of a group $G$ is commensurated if the commensurator of $Q$ in $G$ is the entire group $G$. Our main result is that a finitely generated group $G$ containing an infinite, finitely generated, commensurated subgroup $H$ of infinite index in $G$ is one-ended and semistable at $\infty$. Furthermore, if $Q$ and $G$ are finitely presented and either $Q$ is one-ended or the pair $(G, Q)$ has one filtered end, then $G$ is simply connected at $\infty$. A normal subgroup of a group is commensurated, so this result is a generalization of M Mihalik's result [17] and of B Jackson's result [11]. As a corollary, we give an alternate proof of V M Lew's theorem that a finitely generated group $G$ containing an infinite, finitely generated, subnormal subgroup of infinite index is semistable at $\infty$. So several previously known semistability and simple connectivity at $\infty$ results for group extensions follow from the results in this paper. If $\phi: H \rightarrow H$ is a monomorphism of a finitely generated group and $\phi(H)$ has finite index in $H$, then $H$ is commensurated in the corresponding ascending HNN extension, which in turn is semistable at $\infty$.
\end{abstract}

20F69; 20F65

\section{Introduction}

Given a group $G$ and a subgroup $H$ of $G$, the element $g$ of $G$ is in the commensurator of $H$ in $G$ (denoted $\operatorname{Comm}(H, G)$ ) if $g_{H g}^{-1} \cap H$ has finite index in both $H$ and $g \mathrm{Hg}^{-1}$. In the mid-1960s, A Borel [1] proved a series of results that highlight the critical nature of commensurators in the structure of semisimple Lie groups. These results were extended by G A Margulis [16] in 1975. If $G$ is the commensurator of $Q$ in $G$, then $Q$ is commensurated in $G$. In particular, if $H$ is normal in $G$, then $H$ is commensurated in $G$. The authors [3], develop the basic theory of commensurated subgroups and showed this theory closely parallels the theory of normal subgroups of a group, but with subtle differences.

A locally finite, connected CW-complex $X$ is semistable at $\infty$ if any two proper maps $r, s:[0, \infty) \rightarrow X$ that converge to the same end are properly homotopic. The early ideas 
of R Lee and F Raymond [14], and FE A Johnson [12], on the "fundamental group of an end" were instrumental in extending the idea of semistability at $\infty$ of a space to the notion of the semistability at $\infty$ for a finitely presented group. R Geoghegan [7] introduced the idea of using shape theory as the correct setting to study the homology of ends of groups. The best reference for the fundamentals of the subject of semistability at $\infty$ is R Geoghegan's book [8]. Many classes of finitely generated groups are known to be semistable at $\infty$ (see Mihalik $[17 ; 19 ; 20 ; 21 ; 22]$, for instance). It is unknown if all finitely presented groups are semistable at $\infty$. If a finitely presented group $G$ is semistable at $\infty$, then one can define invariants for $G$, such as the fundamental group at an end of $G$, independent of choice of basepoint ray in some associated space. The idea of semistability at $\infty$ is also of interest in the study of cohomology of groups. $\mathrm{R}$ Geoghegan and M Mihalik [9], have shown that if the group $G$ is finitely presented and semistable at $\infty$, then $H^{2}(G ; \mathbb{Z} G)$ is free abelian. It should be noted that a basic unsolved problem in the study of group cohomology is whether or not $H^{2}(G ; \mathbb{Z} G)$ is free abelian for all finitely presented groups $G$.

The study of ends of groups was started by H Freudenthal [6] and H Hopf [10]. A finitely generated group $G$ has either $0,1,2$ or an infinite number of ends. It is elementary to see that finitely presented groups with either 0 or 2 ends are semistable at $\infty$. By Mihalik [21], and Dunwoody's accessibility theorem [5], the semistability question for finitely presented groups reduces to the question of whether or not all one-ended finitely presented groups are semistable at $\infty$.

The strongest result to date in this subject is the following combination result of M Mihalik and S Tschantz [24].

Theorem 1.1 (M Mihalik, S Tschantz) If $G=A *_{H} B$ is an amalgamated product where $A$ and $B$ are finitely presented and semistable at $\infty$ and $H$ is finitely generated, then $G$ is semistable at $\infty$. If $G=A *_{H}$ is an HNN-extension where $A$ is finitely presented and semistable at $\infty$ and $H$ is finitely generated, then $G$ is semistable at $\infty$.

This result generalizes to the obvious statement about graphs of groups and was used by Mihalik and Tschantz [23] to prove that all one relator groups are semistable at $\infty$. It should be noted that this result is non-trivial when $A$ and $B$ are free groups.

All word-hyperbolic groups are semistable at $\infty$ (see G Swarup [28]). R Geoghegan [7] has shown that a one-ended CAT(0) group $G$ is semistable at $\infty$ if and only if some (equivalently, any) visual boundary for $G$ has the shape of a locally connected continuum. It is elementary to construct a semistable at $\infty$, one-ended CAT( 0$)$ group with non-locally connected boundary. For instance, the direct product of the integers with the free group of rank 2 has visual boundary homeomorphic to the suspension of 
a Cantor set. While the suspension of a Cantor set is non-locally connected, it has the same shape as the Hawaiian earring, which is a locally connected space. Mihalik [20] defined a notion of semistability at $\infty$ for a finitely generated group that generalizes the original definition (ie a finitely presented group is semistable at $\infty$ with respect to the alternative definition if and only if it is semistable at $\infty$ with respect to the original definition). With this more general definition, the finitely generated analogs to the main results obtained in $[17 ; 19]$ are quite apparent. In fact, this more general definition is used to show certain finitely presented groups are semistable at $\infty$ (see [20]). In his $\mathrm{PhD}$ dissertation, Lew [15] proved that if $G$ is a finitely generated group containing an infinite, finitely generated, subnormal subgroup $H$ of infinite index in $G$, then $G$ is one-ended and semistable at $\infty$. Lew's proof of this theorem generalized arguments used in the proofs in [17] and [19]. Our main theorem is used in Section 5 to produce an alternative proof of Lew's theorem.

Theorem 1.2 (Main theorem) If a finitely generated group $G$ has an infinite, finitely generated, commensurated subgroup $Q$, and $Q$ has infinite index in $G$, then $G$ is oneended and semistable at $\infty$. Furthermore, if $G$ and $Q$ are finitely presented and either $Q$ is one-ended or the pair $(G, Q)$ has one filtered end, then $G$ is simply connected at $\infty$.

As an example, the cyclic subgroup $\langle x\rangle$ of the Baumslag-Solitar group

$$
B(m, n) \equiv\left\langle x, t: t^{-1} x^{m} t=x^{n}\right\rangle \quad \text { (for non-zero integers } m, n \text { ) }
$$

is commensurated in $B(m, n)$.

A connected CW-complex $X$ is simply connected at $\infty$ if for each compact set $C$ in $X$ there is a compact set $D$ in $X$ such that loops in $X-D$ are homotopically trivial in $X-C$. Simple connectivity at $\infty$ implies semistability at $\infty$. As with semistability at $\infty$, the idea of simple connectivity at $\infty$ can be extended from spaces to finitely presented groups and if $G$ is finitely presented and simply connected at $\infty$, then $H^{2}(G ; \mathbb{Z} G)$ is trivial. L Siebenmann [26] developed the idea of simple connectivity at $\infty$ to give an obstruction to finding a boundary for an open manifold. R Lee and F Raymond [14] used the idea of the simple connectivity at $\infty$ of a group in order to analyze manifolds covered by Euclidean space. B Jackson [11] proves:

Theorem 1.3 (B Jackson) If $1 \rightarrow H \rightarrow G \rightarrow K \rightarrow 1$ is a short exact sequence of infinite, finitely presented groups and either $H$ or $K$ is one-ended, then $G$ is simply connected at $\infty$.

In his thesis, J Profio [25] improved Jackson's result: 
Theorem 1.4 (J Profio) Suppose $H \triangleleft N \triangleleft G$ where $G$ and $H$ are finitely presented, $H$ is 1 -ended and $H$ has infinite index in $G$. Then $G$ is simply connected at $\infty$.

M Davis [4] constructs examples of aspherical closed $n$-manifolds for $n \geq 4$ that are not covered by $\mathbb{R}^{n}$. In fact, Davis argues that the fundamental groups of his manifolds are semistable at $\infty$, but not simply connected at $\infty$ (and hence not covered by $\mathbb{R}^{n}$ ). All of Davis's groups are subgroups of finite index in finitely generated Coxeter groups. Mihalik [22] showed all Artin and Coxeter groups are semistable at $\infty$.

The remainder of the paper is organized as follows. In Section 2 we give the basics of commensurated subgroups of groups. This includes a geometric characterization of commensurated groups and a result connecting the filtered ends of a Cayley graph of a group to the ends of a Schreier coset graph. In Section 3, we say what it means for a finitely generated group to be semistable at $\infty$ and list a number of equivalent formulations of this concept. The proof of the main theorem takes up most of Section 4. Section 5 is devoted to an alternative proof of a theorem of V M Lew. Section 6 contains a generalization of a result of B Jackson about simply connected at $\infty$ group extensions. The techniques of proof in sections 4, 5 and 6 are geometric. The working definition of commensurated subgroup is used to construct proper homotopies between proper edge path rays in certain Cayley 2-complexes and to homotopically kill certain loops by homotopies that avoid prescribed compact sets.

\section{Commensurable preliminaries}

If $S$ is a finite generating set for a group $G, \Gamma(G, S)$ the Cayley graph of $G$ with respect to $S$ and $H$ a subgroup of $G$, then for any $g_{1}, g_{2} \in G$, the Hausdorff distance between $g_{1} H$ and $g_{2} H$, denoted $D_{S}\left(g_{1} H, g_{2} H\right)$, is the smallest integer $K$ such that for each element $h$ of $H$ the edge path distance from $g_{1} h$ to $g_{2} H$ in $\Gamma$ is at most $K$ and the edge path distance from $g_{2} h$ to $g_{1} H$ in $\Gamma$ is at most $K$. If no such $K$ exists, then $D_{S}\left(g_{1} H, g_{2} H\right)=\infty$. Conner and Mihalik [3] prove the following geometric characterization of commensurated subgroups of finitely generated groups. This characterization is the working definition of commensurated subgroup in this paper.

Proposition 2.1 (G Conner, M Mihalik) Suppose $S$ is a finite generating set for a group $G$ and $H$ is a subgroup of $G$. Then $g \in G$ is in $\operatorname{Comm}(H, G)$ if and only if the Hausdorff distance $D_{S}(H, g H)<\infty$ if and only if $D_{S}\left(H, g \mathrm{Hg}^{-1}\right)<\infty$.

In particular, a subgroup $Q$ of a finitely generated group $G$ is commensurated in $G$ if and only if the Hausdorff distance $D_{S}(Q, g Q)$ is finite for all $g \in G$ if and only if $D_{S}\left(Q, g Q g^{-1}\right)$ is finite for all $g \in G$. 
Suppose $G$ is a group with finite generating set $S$ and $H$ is a subgroup of $G$. Let $\Lambda(S, H, G)$ be the graph with vertices the left cosets $g H$ of $G$ and a directed edge (labeled $s$ ) from $g H$ to $f H$ if for some $s \in S$ and $h_{1}, h_{2} \in H$, we have $g h_{1} s h_{2}=f$. (Equivalently, in the Cayley graph $\Gamma(S, G)$, there is an edge labeled $s$ with initial point in $g H$ and end point in $f H$.) Basically, $\Lambda$ is a (left) Schreier coset graph. Note that $\Lambda$ may have several edges labeled $s$ at a vertex.

The following result appears in [3] and is used in the proof of our main theorem.

Proposition 2.2 (G Conner, M Mihalik) Suppose $G$ is a group with finite generating set $S$ and $Q$ is commensurated in $G$. Then the graph $\Lambda(S, Q, G)$ is locally finite and $G$ acts (on the left) transitively on the vertices of $\Lambda$ and by isometries (using the edge path metric) on $\Lambda$. For the Cayley graph $\Gamma(S, G)$ of $G$, the projection map $p: \Gamma(S, G) \rightarrow \Lambda(S, Q, G)$ respects the action of $G$ and induces a bijection from the filtered ends of $\Gamma(S, G)$ to the ends of $\Lambda(S, Q, G)$. The graph $\Lambda(S, Q, G)$ has 0,1 , 2 or infinitely many ends.

\section{Semistability preliminaries}

Much of the groundwork for studying the notion of semistability for a finitely presented group has appeared in $[11 ; 12 ; 13 ; 14 ; 17]$, and is well-organized in [7]. We will recall some of the ideas presented in these papers to set the notation for future use.

A continuous function $f: X \rightarrow Y$ is proper if for each compact subset $C$ of $Y$, $f^{-1}(C)$ is compact in $X$. A proper map $r:[0, \infty) \rightarrow X$ is called a ray in $X$. If $K$ is a locally finite, connected CW-complex, then one can define an equivalence relation $\sim$ on the set $A$ of all rays in $K$ by setting $r \sim s$ if and only if for each compact set $C \subset K$, there exists an integer $N(C)$ such that $r([N(C), \infty))$ and $s([N(C), \infty))$ are contained in the same unbounded path component of $K-C$ (a path component of $K-C$ is unbounded if it is not contained in any compact subset of $K$ ). An equivalence class of $A / \sim$ is called an end of $K$, the set of equivalence classes of $A / \sim$ is called the set of ends of $K$ and two rays in $K$, in the same equivalence class, are said to converge to the same end. The cardinality of $A / \sim$, denoted by $e(K)$, is the number of ends of $K$.

If $\mathrm{G}$ is a finitely generated group with generating set $S$, then denote the Cayley graph of $G$ with respect to $S$ by $\Gamma(G, S)$. We define the number of ends of $G$, denoted by $e(G)$, to be the number of ends of the Cayley graph of $G$ with respect to a finite generating set. (In particular, $e(G)=e(\Gamma(G, S)$.) This definition is independent of the choice of finite generating set for $G$. If $G$ is finitely generated, then $e(G)$ is either 
$0,1,2$ or is infinite (in which case it has the cardinality of the real numbers). We let $*$ denote the basepoint of $\Gamma(G, S)$, which corresponds to the identity of $G$.

If $f$ and $g$ are rays in $K$, then one says that $f$ and $g$ are properly homotopic if there is a proper map $H:[0,1] \times[0, \infty) \rightarrow K$ such that $\left.H\right|_{\{0\} \times[0, \infty)}=f$ and $\left.H\right|_{\{1\} \times[0, \infty)}=g$. If $f(0)=g(0)=v$, one says $f$ and $g$ are properly homotopic relative to $v$ (or $\operatorname{rel}\{v\}$ ) if additionally $\left.H\right|_{[0,1] \times\{0\}}=v$.

Definition 3.1 A locally finite, connected CW-complex $K$ is semistable at $\infty$ if any two rays in $K$ converging to the same end are properly homotopic.

Mihalik [17, Theorem 2.1; 19, Lemma 9] provided several equivalent notions of semistability. The space considered in [17] is simply connected, but simple connectivity is not important in that argument. A slight modification of the proofs give the following result.

Theorem 3.2 Suppose $K$ is a locally finite, connected and one-ended $C W$-complex. Then the following are equivalent:

(1) $K$ is semistable at $\infty$.

(2) For any ray $r:[0, \infty) \rightarrow K$ and compact set $C$, there is a compact set $D$ such that for any third compact set $E$ and loop $\alpha$ based on $r$ and with image in $K-D, \alpha$ is homotopic $\operatorname{rel}\{r\}$ to a loop in $K-E$, by a homotopy with image in $K-C$.

(3) For any compact set $C$ there is a compact set $D$ such that if $r$ and $s$ are rays based at $v$ and with image in $K-D$, then $r$ and $s$ are properly homotopic $\operatorname{rel}\{v\}$ by a proper homotopy in $K-C$.

If $K$ is simply connected, then a fourth equivalent condition can be added to this list:

(4) If $r$ and $s$ are rays based at $v$, then $r$ and $s$ are properly homotopic $\operatorname{rel}\{v\}$.

Example 3.3 Note that the one-ended CW-complex obtained by attaching a loop at 0 to the interval $[0, \infty)$ is semistable at $\infty$. Consider a ray $r$ that maps $[0, \infty)$ homeomorphically to $[0, \infty)$ and a ray $s$ that maps $[0,1]$ once around the loop and then maps $[1, \infty)$ homeomorphically to $[0, \infty)$. Clearly $r$ and $s$ are properly homotopic, but not by a proper homotopy $\operatorname{rel}\{0\}$.

The following fact is proved by FE A Johnson $[12 ; 13]$. 
Theorem 3.4 Suppose $X$ and $Y$ are finite, connected $C W$-complexes with $\pi_{1}(X)$ isomorphic to $\pi_{1}(Y)$. Then the universal cover of $X$ is semistable at $\infty$ if and only if the universal cover of $Y$ is semistable at $\infty$.

Definition 3.5 If $G$ is a one-ended, finitely presented group and $X$ is some (equivalently any) finite, two-dimensional CW-complex with fundamental group $G$, then we say $G$ is semistable at $\infty$ if the universal cover of $X$ is semistable at $\infty$.

We now define the notion of semistability for a finitely generated group following Mihalik [20]. We give the definition for one-ended groups since this is the case that concerns us. Suppose $G$ is a one-ended finitely generated group with generating set $S \equiv\left\{g_{1}, g_{2}, \ldots, g_{n}\right\}$ and let $\Gamma(G, S)$ be the Cayley graph of $G$ with respect to this generating set. Suppose $\left\{\alpha_{1}, \alpha_{2}, \ldots, \alpha_{m}\right\}$ is a finite set of relations in $G$ written in the letters $\left\{g_{1}^{ \pm}, g_{2}^{ \pm}, \ldots, g_{n}^{ \pm}\right\}$. For any vertex $v \in \Gamma(G, S)$, there is an edge path cycle labeled $\alpha_{i}$ at $v$. The two dimensional CW-complex $\Gamma_{(G, S)}\left(\alpha_{1}, \ldots, \alpha_{m}\right)$ is obtained by attaching, to each vertex of $\Gamma(G, S), 2$-cells corresponding to the relations $\alpha_{1}, \ldots, \alpha_{n}$.

Mihalik [20] shows that if $S$ and $T$ are finite generating sets for the group $G$ and there are finitely many $S$-relations $P$ such that $\Gamma_{(G, S)}(P)$ is semistable at $\infty$, then there are finitely many $T$-relations $Q$ such that $\Gamma_{(G, T)}(Q)$ is semistable at $\infty$. Hence the following definition:

Definition 3.6 We say $G$ is semistable at $\infty$ if for some finite generating set $S$ for $G$ and finite set of $S$-relations $P$, the complex $\Gamma_{(G, S)}(P)$ is semistable at $\infty$.

Note that if $G$ has finite presentation $\langle S: P\rangle$, then $G$ is semistable at $\infty$ with respect to Definition 3.5 if and only if $G$ is semistable at $\infty$ with respect to Definition 3.6 if and only if $\Gamma_{(G, S)}(P)$ is semistable at $\infty$.

Lemma 3.7 [20, Lemma 2] Suppose the finitely generated group $G$ is one-ended and semistable at $\infty$. If $S$ is a finite generating set for $G$ and $P$ is a finite set of $S$-relations in $G$ such that $\Gamma_{(G, S)}(P)$ is semistable at $\infty$, then there is a finite set $Q$ of $S$-relations such that if $r$ and $s$ are rays in $\Gamma_{(G, S)}(P \cup Q)$ with $r(0)=s(0)$, then $r$ is properly homotopic to $s \operatorname{rel}\{r(0)\}$.

Remark 3.8 Using the third equivalent notion of semistability in Theorem 3.2, it can be shown that in fact the set of relations $Q$ in the previous lemma are unnecessary in order to draw the same conclusion. If $\Gamma_{(G, S)}(P)$ is semistable at $\infty$, and $r$ and $s$ are rays in $\Gamma_{(G, S)}(P)$ with $r(0)=s(0)$, then $r$ is properly homotopic to $s \operatorname{rel}\{r(0)\}$. 
By an edge path ray in a locally finite, connected CW-complex $K$, we mean a proper map $r:[0, \infty) \rightarrow K$ such that for each positive integer $n,\left.r\right|_{[n-1, n]}$ is a homeomorphism to an edge of $K$. If $G$ is finitely generated with finite generating set $S$, then any edge path ray $r:([0, \infty),\{0\}) \rightarrow(\Gamma(G, S), *)$ can be represented as $\left(e_{1}, e_{2}, \ldots\right)$ at $*$ with $e_{i} \in S^{ \pm}$, and $e_{i}$ the label of the $i^{\text {th }}$ edge of $r$. Any edge path $\left(e_{1}, e_{2}, \ldots, e_{k}\right)$ of $\Gamma(G, S)$ corresponds to some group element $e_{1}^{\prime} e_{2}^{\prime} \cdots e_{k}^{\prime}$, where $e_{i}^{\prime} \in S^{ \pm}$. But determining an edge path in $\Gamma(G, S)$ from some word $e_{1}^{\prime} e_{2}^{\prime} \cdots e_{k}^{\prime}$ requires a specified basepoint, since the path $\left(e_{1}^{\prime}, e_{2}^{\prime}, \ldots, e_{k}^{\prime}\right)$ at a vertex $v$ determines a different edge path than $\left(e_{1}^{\prime}, e_{2}^{\prime}, \ldots, e_{k}^{\prime}\right)$ based at another vertex $w$. The action of the group element $w v^{-1}$ on $\Gamma(G, S)$ maps the edge path at $v$ to the edge path at $w$ with the same labeling. If $A$ is a subcomplex of a locally finite, connected CW-complex $K$, let $\operatorname{St}(A)$ denote the subcomplex of $K$ consisting of the union of all $1-$ cells of $K$ that intersect $A$ along with any $n$-cell all of whose vertices lie in $\operatorname{St}(A)$. Note then that $A \subseteq \operatorname{St}(A)$ and if $A$ is a finite subcomplex, then $\operatorname{St}(A)$ is a finite subcomplex by the local finiteness of $K$. We recursively define the $N^{\text {th }}$ star of $A$ for $N=1,2,3, \ldots$ by $S t^{N}(A)=\operatorname{St}\left(S t^{N-1}(A)\right)$, where $S t^{0}(A)=A$. When it is not clear what the over-complex might be, we use the notation $\operatorname{St}(A, K)$ to denote the star of $A$ in $K$.

Since any ray $r:[0, \infty) \rightarrow K$ is properly homotopic to an edge path ray, we may concentrate on edge path rays when dealing with the semistability of a complex.

If $e$ is an edge in $K$ and $\left(e_{1}, e_{2}, e_{3}, \ldots\right)$ is an edge path in $K$ based at the terminal point of $e$, then one denotes by $e *\left(e_{1}, e_{2}, e_{3}, \ldots\right)$ the edge path given by $e$ followed by $\left(e_{1}, e_{2}, e_{3}, \ldots\right)$.

Definition 3.9 For a group $G$ with finite generating set $S$ and a subset $T$ of $S$, we say an edge path in $\Gamma(G, S)$ is a $T$-path if each edge of the path is labeled by an element of $T^{ \pm}$. If the path is infinite and proper, we call it a $T$-ray.

\section{Proof of semistability in the main theorem}

We prove a more general one-ended result than that stated in our main result.

Proposition 4.1 Suppose $A$ is a finitely generated, infinite subgroup of infinite index in a finitely generated group $G$ and $g \mathrm{Ag}^{-1} \cap A$ is infinite for all $g \in G$. Then $G$ is one-ended.

Proof Suppose $S$ is a finite set of generators for $G$ containing a generating set $S_{A}$ for $A$. Let $\Gamma$ be the Cayley graph of $G$ with respect to $S$ and let $\Gamma_{A}$ be the Cayley graph of $A$ with respect to $S_{A}$. We consider $\Gamma_{A}$ to be a subset of $\Gamma$ containing $*$, the 
identity vertex. Let $C$ be a finite subcomplex of $\Gamma$. List elements $g_{1}, \ldots, g_{n}$ of $G$ such that $g_{i} A \neq g_{j} A$ for $i \neq j$ and $g \Gamma_{A} \cap C \neq \varnothing$ if and only if $g A=g_{i} A$ for some $i \in\{1, \ldots, n\}$. Choose $g_{0}$ such that $g_{0} \Gamma_{A} \cap C=\varnothing$. Let $D$ be a finite subcomplex of $\Gamma$ containing $C$ and all bounded components of $g_{i} \Gamma_{A}-C$ for all $i \in\{1, \ldots, n\}$.

It suffices to show that any vertex of $\Gamma-D$ can be joined by a path in $\Gamma-C$ to $g_{0}$. Suppose $v$ is a vertex of $\Gamma-D$.

First we consider the case $v \Gamma_{A} \cap C=\varnothing$. Choose $y$ (in the infinite set) $\left(v \Gamma_{A} v^{-1}\right) \cap$ $\left(g_{0} \Gamma_{A} g_{0}^{-1}\right)$ such that $d(y, C)>\max \left\{|v|,\left|g_{0}\right|\right\}$. Then there are paths from $y$ to $v \Gamma_{A}$ and from $y$ to $g_{0} \Gamma_{A}$ avoiding $C$. Hence there is a path from $v$ to $g_{0}$ avoiding $C$ and the first case is finished.

Next suppose $v \Gamma_{A} \cap C \neq \varnothing$. Then $v$ is in an unbounded component $K$ of $v \Gamma_{A}-C$. Let $N=\max \left\{\left|g_{0}\right|, \ldots,\left|g_{n}\right|\right\}$. Choose $k$ a vertex of $K$ such that $d(k, C)>N$. Then there are paths from $v$ to $k$ and from $k$ to $k g_{i}$ for each $i \in\{0, \ldots, n\}$, all avoiding $C$. At least one of $k g_{i} A$ does not intersect $C$, so by the first case we can connect $v$ to $g_{0}$ avoiding $C$.

For the remainder of the proof of the main theorem, $\mathcal{Q}=\left\{q_{1}, q_{2}, \ldots, q_{n}\right\}$ is a finite generating set for $Q$ and $S=\left\{q_{1}, q_{2}, \ldots, q_{n}, k_{1}, k_{2}, \ldots, k_{t}\right\}$ is a generating set for $G$ where $k_{i} \notin Q$. Let $\mathcal{K}=\left\{k_{1}, \ldots, k_{t}\right\}$. Our hypothesis states that for each $g \in G$, the Hausdorff distance between $Q$ and $g Q$ is finite in $\Gamma(G, S)$.

Consider the left (Scherier) coset graph $\Lambda(S, Q, G)$ with vertex set, the set of all cosets $g Q$ in $G$. A directed edge labeled $s$ will have initial vertex $g_{1} Q$ and terminal vertex $g_{2} Q$ if there is an edge labeled $s$ in $\Gamma(G, S)$ beginning in $g_{1} Q$ and ending in $g_{2} Q$. By Proposition 2.2, $\Lambda(S, Q, G)$ is locally finite. There is a quotient map $\rho: \Gamma(G, S) \rightarrow \Lambda(S, Q, G)$ respecting the left action of $G$ on these graphs, such that each edge labeled by an element of $Q$ is mapped to a point.

Lemma 4.2 Suppose $S$ is a finite generating set for the group $G$ and $Q$ is a finitely generated commensurated subgroup of $G$ (with generating set a subset of $S$ ). There is an integer $F$ such that if $g Q$ and $h Q$ are distinct cosets (vertices) of $\Lambda(S, Q, G)$ connected by an edge labeled $s \in S^{ \pm 1}$, then for each $v \in g Q \subset \Gamma(S, G)$ there is a $Q$-path $\alpha$ at $v$ in $\Gamma(S, G)$ of length less than $F$ such that the path $(\alpha, s)$ ends in $h Q$.

In particular: Suppose $\alpha \equiv\left(e_{1}, e_{2}, \ldots\right)$ is an edge path (possibly infinite) at $v \in$ $\Lambda(S, Q, G)$ (with $i^{\text {th }}$ edge labeled $\left.e_{i}\right)$ and $v^{\prime}$ is a vertex of $\Gamma(G, S)$ such that $\rho\left(v^{\prime}\right)=v$ (equivalently $v^{\prime} Q=v$ ). Then there is an edge path $\alpha^{\prime} \equiv\left(\alpha_{0}^{\prime}, e_{1}, \alpha_{1}^{\prime}, e_{1}, \ldots\right)$ at $v^{\prime}$ with $\alpha_{i}^{\prime}$ a $Q$-edge path of length less than $F$ such that the edge path (determined by) $\rho \alpha^{\prime}$ is $\alpha$, ie there is a $(Q, F)$-“approximate" path lifting for $\rho$. 
Proof Suppose $v \in g Q$ and the edge labeled $s$ at $v$ ends in $h Q$. By translation, we assume $v=1 \in G, g=1$ and $h=s$. As $Q$ is commensurated in $G, s Q s^{-1} \cap Q$ has finite index in $Q$. Hence there is an integer $F_{S}$, such that for any vertex $w \in Q$, there is a $Q$-edge path in $\Gamma(S, G)$ of length less than $F_{s}$ from $w$ to a vertex $w^{\prime}$ of $Q \cap s Q s^{-1}$. As $w^{\prime} \in s Q s^{-1}, w^{\prime} s \in s Q$, ie the edge labeled $s$ at $w^{\prime}$ ends in $s Q$. Let $F=\max \left\{F_{s}\right\}_{s \in S^{ \pm 1}}$.

Remark 4.3 For $\alpha$ and $\alpha^{\prime}$ as in Lemma 4.2, we call $\alpha^{\prime}$ a $(Q, F)$-approximate lift of $\alpha$. Note that Lemma 4.2 does not imply that if $v$ and $w$ are vertices of the same coset $u Q$, then there are approximate lifts of a path $\alpha$ at $\rho(v) \in \Lambda(S, Q, G)$ to $v$ and $w$ that are $G$ translates of one another in $\Gamma(G, S)$.

The next lemma basically has the same proof as [19, Lemma 3].

Lemma 4.4 For each vertex $v$ of $\Lambda(S, Q, G)$, there is an edge path ray $s_{v}$ at $v$, such that for any finite subgraph $C$ of $\Lambda(S, Q, G)$ only finitely many $s_{v}$ intersect $C$. Furthermore, if $w \in v \equiv w Q$, and we let $s_{w}$ be a $(Q, F)$-approximate lift of $s_{\rho(w)}$ to $w \in \Gamma(G, S)$, then:

(i) For any finite subgraph $D$ of $\Gamma(G, S)$ there are only finitely many vertices $w \in \Gamma(G, S)$ such that $s_{w}$ intersects $D$ non-trivially.

(ii) For any $w \in G$, only finitely many vertices $z$ of $s_{w}$ are such that $z Q$ intersects $D$ non-trivially.

Proof If $\mathcal{G}$ is a locally finite, infinite graph, then for each vertex $v$ of $\mathcal{G}$ there is an edge path ray $s_{v}$ at $v$ such that for any finite subgraph $C$ of $\mathcal{G}$ only finitely many $v$ are such that $s_{v}$ intersects $C$. (The idea is this: Choose a base vertex $x$. For any integer $n>0, \mathcal{G}-S t^{n}(x)$ has only finitely many components. For the finitely many vertices $v$ in $S t(x)$ or a bounded component of $\mathcal{G}-S t(x)$, choose $s_{v}$ to be an arbitrary edge path ray at $v$. If $v$ is a vertex of $S t^{2}(x)$ or of a bounded component of $\mathcal{G}-S t^{2}(x)$, and $s_{v}$ is not defined, then $v$ belongs to an unbounded component of $\mathcal{G}-\operatorname{St}(x)$. Choose $s_{v}$ to be an edge path ray at $v$ in $\mathcal{G}-S t(x)$. Continue in this fashion.) Now pick such edge path rays for the vertices of $\Lambda(S, Q, G)$.

As $\rho\left(s_{w}\right)=s_{\rho(w)}, s_{w}$ intersects $D$ if and only if $s_{\rho(s)}$ intersects $\rho(D)$. Hence we may finish the proof of (i) by showing at most finitely many vertices $v$ of a coset $g Q$ are such that $s_{v}$ intersects $D$. Otherwise there are infinitely many distinct vertices $v_{1}, v_{2}, \ldots$ in $g Q \subset \Gamma(G, S)$ such that each edge path ray $s_{v_{i}}$ passes through the vertex $d$ of $D$. In $\Lambda(S, Q, G)$, write the edge path ray $s_{g} Q \equiv\left(e_{1}, e_{2}, \ldots\right)$. By Lemma 4.2, we may write $s_{v_{i}}=\left(\alpha_{i, 1}, e_{1}, \alpha_{i, 2}, e_{2}, \ldots\right)$ in $\Gamma(G, S)$, where $\alpha_{i, j}$ is a $Q$-edge path of length 
less than $F$. Let $n(i)$ be such that some vertex of $\alpha_{i, n(i)}$ is $d$. Since the $v_{i}$ are distinct and the length of each $\alpha_{i, j}$ is less than $F$, the sequence of integers $\{n(1), n(2), \ldots\}$ is unbounded. But then the initial vertex of $e_{n(i)}$ (on $\left.s_{g Q} \equiv\left(e_{1}, e_{2}, \ldots\right)\right)$ is $\rho(d)$. This is impossible since $s_{g} Q \equiv\left(e_{1}, e_{2}, \ldots\right)$ is proper, and (i) is proved.

Part (ii) follows immediately from the fact that $\rho\left(s_{w}\right)=s_{\rho(w)}$ is a proper map.

By Lemma 4.2, if two distinct cosets $g_{1} Q$ and $g_{2} Q$ of $G$ are connected by an edge in $\Gamma(G, S)$, then they are of Hausdorff distance at most $F$. Choose $M$ such that if two vertices of $Q$ in $\Gamma(G, S)$ are within $2 F+1$ of one another, then their $\mathcal{Q}$-distance is at most $M$. Let $P$ be the set of all $S$-relations in $G$ of length at most $2 F+1+M$. Let $\widetilde{\Gamma}$ be $\Gamma_{(G, S)}(P)$.

Lemma 4.5 [19, Lemma 2] At each vertex $v$ of $\Gamma(G, S)$ there exists a $\mathcal{Q}$-ray $q_{v}$, such that for any finite subcomplex $C$ in $\Gamma(G, S)$ there are only finitely many vertices $v$ such that $q_{v}$ meets $C$.

For each $S$-relation $r$ of $G$, consider the $\mathcal{K}$-word $r_{\mathcal{K}}$ obtained by eliminating from $r$, the $\mathcal{Q}$-letters (and their inverses). If $v$ is a vertex of $\Gamma(G, S)$ and $\alpha$ the edge path loop corresponding to $r$ at $v$, then $\rho(\alpha)$ (in $\Lambda(S, Q, G)$ ) has labeling $r_{\mathcal{K}}$. Let $\widetilde{\Lambda}(S, Q, G)$ be the $2-$ complex obtained from $\Lambda(S, Q, G)$ by attaching a $2-$ cell to each loop $\rho r$ (with label $r_{\mathcal{K}}$ ) where $r$ is a loop of $\Gamma(G, S)$ of length at most $2 F+M+1$ (only one 2-cell for a given such loop in $\Lambda(S, Q, G)$ ). Then $\tilde{\Lambda}(S, Q, G)$ is locally finite and there is a natural map $\tilde{\rho}: \widetilde{\Gamma}(G, S) \rightarrow \widetilde{\Lambda}(S, Q, G)$ extending $\rho$ and respecting the action of $G$.

Lemma 4.6 If $k \in \mathcal{K}^{ \pm}$labels an edge of $\tilde{\Gamma}$ from $v$ to $w$ and $r=\left(e_{1}, e_{2}, e_{3}, \ldots\right)$ is a $\mathcal{Q}$-ray at $v$, then $r$ is properly homotopic $\operatorname{rel}\{v\}$ to $k *\left(f_{1}, f_{2}, \ldots\right)$, where $\left(f_{1}, f_{2}, \ldots\right)$ is a $\mathcal{Q}$-ray at $w$, by a homotopy $H$ whose image is a subset of

$$
S t S t^{2 F+M+1}(\operatorname{Im}(r), \widetilde{\Gamma}),
$$

and the image of $\tilde{\rho} \circ H$ is a subset of the finite complex $\operatorname{St}(\widetilde{\rho}(k))$.

Proof Let $v_{i}$ be the terminal vertex of $e_{i}$. Let $v_{0}=v, w_{0}=w, \alpha_{0}$ be the empty path. For each $i \geq 1$, Lemma 4.2 implies there is a $\mathcal{Q}$-edge path $\alpha_{i}$ of length less than $F$ at $v_{i}$ so that $\left(\alpha_{i}, k\right)$ ends at $w_{i} \in k Q$. Note that in $\widetilde{\Gamma}$ the distance from $w_{i}$ to $w_{i+1}$ is at most $2 F+1$. For $i \geq 1$, let $f_{i}$ be a $\mathcal{Q}$-edge path in $\widetilde{\Gamma}$ of length at most $M$ from $w_{i-1}$ to $w_{i}$. The loop $\left(\alpha_{i}, k, f_{i+1}, k^{-1} \alpha_{i+1}^{-1}, e_{i+1}^{-1}\right)$ has length at most $2 F+1+M$ and so bounds a $2-$ cell of $\widetilde{\Gamma}$. Hence $\left(e_{1}, e_{2}, \ldots\right)$ is properly homotopic to $k *\left(f_{1}, f_{2}, \ldots\right)$ by a homotopy $H$ with image in $\left.S t^{2 F+1+M}(\operatorname{Im}(r)), \widetilde{\Gamma}\right)$. As each $\alpha_{i}$ and each $f_{i}$ is a $\mathcal{Q}$-word, $\tilde{\rho} \circ H$ has image in $\operatorname{St}(\tilde{\rho}(k))$. 
Recall, for each vertex $v \in \widetilde{\Gamma}, s_{v}$ is a $(Q, F)$-approximate lift of $s_{\rho(v)}$ (see Lemma 4.4).

Lemma 4.7 Suppose $D$ is a finite subcomplex of $\widetilde{\Gamma}$. Then there exists a finite complex $E_{1}(D) \subseteq \widetilde{\Gamma}$ such that if $b=\left(e_{1}, e_{2}, e_{3}, \ldots\right)$ is a $\mathcal{Q}$-ray at $v$ with image in $\widetilde{\Gamma}-E_{1}(D)$, then $b$ is properly homotopic $\operatorname{rel}\{v\}$ to $s_{v}$ by a homotopy in $\widetilde{\Gamma}-D$.

Proof Let $L=2 F+M+1$ (the constant of Lemma 4.6). There are only finitely many vertices $w \in \tilde{\Lambda}$ such that the edge path rays $s_{w}$ of Lemma 4.4 intersect $S t(\tilde{\rho}(D))$, nontrivially. Call these vertices $y_{1}, y_{2}, \ldots, y_{l}$. Since each $s_{y_{i}}$ is proper, there are integers $J_{i}$ such that each edge of the ray $s_{y_{i}}$ following the $J_{i}^{\text {th }}$ edge is in $\tilde{\Lambda}-S t(\widetilde{\rho}(D))$. Let $J$ be the maximum $J_{i}$ for $i=1,2, \ldots, l$. By Lemma 4.4, if $w$ is any vertex of $\widetilde{\Gamma}$ and $e$ is the $j^{\text {th }}$ edge of $s_{w}$ for $j>F J$, then $\tilde{\rho}(e)=d$ (or a vertex of $d$ ) for $d$ the $k^{\text {th }}$ edge of $s_{\rho(w)}$ for some $k>J$. By the definition of $J, d$ does not intersect $S t(\widetilde{\rho}(D))$ and so $\tilde{\rho}(e)$ does not intersect $S t(\tilde{\rho}(D))$. In particular:

(*) $\quad$ If $w$ is any vertex of $\widetilde{\Gamma}$ and $e$ is the $j^{\text {th }}$-edge of $s_{w}$ for $j>F J$, then $\tilde{\rho}(e) \subset$ $\widetilde{\Lambda}-S t(\tilde{\rho} D)$.

Let $E_{1}(D)$ be a compact subcomplex of $\widetilde{\Gamma}$ such that $S t^{F J L}(D) \subseteq E_{1}(D)$ and such that $E_{1}(D)$ contains the finite set of vertices $v$ in $\widetilde{\Gamma}$ such that $s_{v}$ intersects $S^{F J L}(D)$. Assume $b$ and $v$ satisfy the hypothesis of the lemma. The edge path ray $s_{v}$ (in $\left.\widetilde{\Gamma}-S t^{F J L}(D)\right)$ has the form $\left(\alpha_{0}, c_{1}, \alpha_{1}, c_{2}, \ldots\right)$, where $\alpha_{i}$ is a $\mathcal{Q}$-path of length less than $F$ and $c_{i}$ is a $\mathcal{K}$-edge. Here $s_{v}$ is a $(Q, F)$-approximate lift of $s_{\rho(v)}=$ $\left(c_{1}^{\prime}, c_{2}^{\prime}, \ldots\right)$ (where $c_{i}^{\prime}$ has the same label as $c_{i}$ ).

Let $v_{i}, w_{i}$ be the initial and terminal vertices of $c_{i}$, respectively. Let $b_{0}$ be the $\mathcal{Q}-$ edge path ray $\left(\alpha_{0}^{-1}, b\right)$. By Lemma $4.6, b_{0}$ is properly homotopic $\operatorname{rel}\left\{v_{1}\right\}$ to $c_{1} * b_{1}$, where $b_{1}$ is a $\mathcal{Q}$-ray at $w_{1}$, by a proper homotopy $H_{1}$ with image in $S t^{L}\left(\operatorname{Im}\left(b_{0}\right)\right)$. In particular, $b_{1}$ has image in $\widetilde{\Gamma}-S t^{(F J-1) L}(D)$. Again by Lemma $4.6,\left(\alpha_{1}^{-1}, b_{1}\right)$ is properly homotopic $\operatorname{rel}\left\{v_{2}\right\}$ to $c_{2} * b_{2}$, where $b_{2}$ is a $\mathcal{Q}$-edge path ray, by a proper homotopy $\mathrm{H}_{2}$ with image in

$$
S t^{L}\left(\operatorname{Im}\left(b_{1}\right)\right) \subset \widetilde{\Gamma}-S t^{(F J-2) L}(D) .
$$

Iterating the above process, the $\mathcal{Q}$-ray $\left(\alpha_{j}^{-1}, b_{j}\right)$ is properly homotopic $\operatorname{rel}\left\{v_{j+1}\right\}$ to $c_{j+1} * b_{j+1}$, where $b_{j+1}$ is a $\mathcal{Q}$-ray, by a proper homotopy $H_{j+1}$ with image in $S t^{L}\left(\operatorname{Im}\left(b_{j}\right)\right)$. Let $H$ be the homotopy of $b$ to $s_{v}$ obtained by patching together these $H_{i}$. For $i \leq F J, H_{i}$ has image in $\widetilde{\Gamma}-D$. By Lemma 4.6, $\widetilde{\rho} \circ H_{j}$ has image in $\operatorname{St}\left(\tilde{\rho}\left(c_{j}\right)\right)$. By $(*)$, if $j>F J$, then $\tilde{\rho}\left(c_{j}\right)$ misses $\operatorname{St}(\tilde{\rho}(D))$. So $\operatorname{St}\left(\widetilde{\rho}\left(c_{j}\right)\right)$ misses $\tilde{\rho}(D)$. For all positive integers $j, H_{j}$ misses $D$ and $H$ misses $D$. 
It remains to show that $H$ is a proper. Let $C \subseteq \widetilde{\Gamma}$ be a finite subcomplex. Since $\widetilde{\rho}\left(s_{v}\right)$ is proper in $\tilde{\Lambda}$, there exists an integer $R$ such that if $j>R$, then $\tilde{\rho}\left(c_{j}\right)$ misses $\operatorname{St}(\tilde{\rho}(C))$. As $\tilde{\rho} \circ H_{j}$ has image in $\operatorname{St}\left(\widetilde{\rho}\left(c_{j}\right)\right), H_{j}$ misses $C$ when $j>R$. Since only finitely many of the proper homotopies $H_{j}$ have image that intersect an arbitrary finite subcomplex $C, H$ is proper.

Lemma 4.8 Suppose $D \subseteq \widetilde{\Gamma}$ is compact. There exists a compact set $E_{2}(D) \subseteq \tilde{\Gamma}$ such that if $e$ is an edge in $\widetilde{\Gamma}-E_{2}(D)$ from $v$ to $w$, then the $\mathcal{Q}$-ray $q_{v}$ is properly homotopic to $e * q_{w} \operatorname{rel}\{v\}$ by a proper homotopy in $\widetilde{\Gamma}-D$.

Proof Again let $L=2 F+M+1$ (the constant of Lemma 4.6). Let $E_{2}(D)$ be a compact subcomplex of $\widetilde{\Gamma}$ containing $S t^{L}\left(E_{1}(D)\right)$ and the finite set of vertices $x$ such that $q_{x}$ intersects $S t^{L}\left(E_{1}(D)\right)$. If $e \in \mathcal{K}^{ \pm 1}$, then by Lemma 4.6, $q_{v}$ is properly homotopic to $e * \beta \operatorname{rel}\{v\}$, where $\beta$ is a $\mathcal{Q}$-ray at $w$ and this homotopy has image in $S t^{L}\left(\operatorname{Im}\left(q_{v}\right)\right)$. In particular, $\beta$ avoids $E_{1}(D)$. By Lemma 4.7, $\beta$ and $q_{w}$ are properly homotopic $\operatorname{rel}\{w\}$ to $s_{w}$ by proper homotopies in $\widetilde{\Gamma}-D$. Combining these homotopies gives the result.

If $e \in \mathcal{Q}^{ \pm 1}$, then Lemma 4.7 implies $q_{v}$ and $e * q_{w}$ are both properly homotopic $\operatorname{rel}(v)$ to $s_{v}$ by a proper homotopy in $\widetilde{\Gamma}-D$. Combining homotopies gives the desired homotopy.

Lemma 4.9 Suppose $s=\left(s_{1}, s_{2}, s_{3}, \ldots\right)$ is an edge path ray at a vertex $v$ in $\widetilde{\Gamma}$, then $s$ is properly homotopic to $q_{v} \operatorname{rel}\{v\}$.

Proof Choose a sequence of compact subcomplexes $\left\{C_{i}\right\}_{i=1}^{\infty}$ such that $\bigcup_{i=1}^{\infty} C_{i}=\widetilde{\Gamma}$, $C_{i}$ is contained in the interior of $C_{i+1}$, and such that $C_{i+1}$ contains $E_{2}\left(C_{i}\right)$. Let $v_{i}$ be the end point of $s_{i}$. Define $H:[0, \infty) \times[0, \infty) \rightarrow \widetilde{\Gamma}$ as follows: If $R$ is the largest integer such that the edge $s_{i}$ misses $C_{R}$, then by definition of $C_{R}, q_{v_{i-1}}$ is properly homotopic rel $\left\{v_{i-1}\right\}$ to $s_{i} * q_{v_{i}}$ by a proper homotopy $H_{i}$, missing $C_{R-1}$. Define $H$ on $[i-1, i] \times[0, \infty)$ to be $H_{i}$.

In order to check that $H$ is proper, it suffices to show that for any compact set $C \subseteq \widetilde{\Gamma}$ only finitely many $H_{j}$ intersect $C$. This follows from the fact that $C \subseteq C_{i}$ for some index $i$. Since $s$ is proper, there is an integer $W(i)$ such that for all $j \geq W_{i}, s_{j}$ lies in $\widetilde{\Gamma}-C_{i+1}$. So, $H_{j}$ avoids $C$ and therefore $H$ is proper.

This completes the semistability part of our main theorem.

If $H$ is a group and $\phi: H \rightarrow H$ is a monomorphism the group with presentation $\left\langle t, H: t^{-1} h t\right.$ for all $\left.h \in H\right\rangle$ is called the ascending HNN extension of $H$ by $\phi$ and 
is denoted $H *_{\phi}$. The main theorem of Mihalik [18] states that if $H$ is a finitely presented group and $\phi: H \rightarrow H$ a monomorphism, then the ascending HNN extension $H *_{\phi}$ is one-ended and semistable at $\infty$. Consider a general finite presentation of the form $\left\langle t, h_{1}, \ldots, h_{n}: r_{1}, \ldots, r_{n}, t^{-1} h_{1} t=w_{1}, \ldots, t^{-1} h_{n} t=w_{n}\right\rangle$, where $r_{i}$ and $w_{i}$ are words in $\left\{h_{1}^{ \pm 1}, \ldots, h_{n}^{ \pm 1}\right\}$ for all $i$. The group $G$ of this presentation is the ascending HNN extension $H *_{\phi}$, where $H$ is generated by $\left\{h_{1}, \ldots, h_{n}\right\}$ and $\phi$ is the monomorphism $\phi: H \rightarrow H, \phi\left(h_{i}\right)=w_{i}$ for all $i$. While $G$ is finitely presented it would seem rare that the finitely generated group $H$ would be finitely presented. It has long been suggested that ascending HNN extensions of this form may be a good place to search for non-semistable at $\infty$, finitely presented groups. Conner and Mihalik [3] show that if $H$ is finitely generated and the image of the monomorphism $\phi: H \rightarrow H$ has finite index in $H$, then $H$ is commensurated in $H *_{\phi}$. As a direct consequence of this result and our main theorem we have:

Corollary 4.10 Suppose $H$ is a finitely generated group and $\phi: H \rightarrow H$ is a monomorphism such that $\phi(H)$ has finite index in $H$. Then, the ascending $H N N$ extension $H *_{\phi}$ is semistable at $\infty$.

\section{A theorem of Lew}

Our goal in this section is to give an alternate proof of a theorem of V M Lew [15].

Theorem 5.1 (V M Lew) Suppose $H$ is an infinite, finitely generated, subnormal subgroup of the finitely generated group $G$ and $H$ has infinite index in $G$. Then $G$ is one-ended and semistable at $\infty$.

Proof Suppose $k>0$ and $H=N_{0} \triangleleft N_{1} \triangleleft N_{2} \triangleleft \cdots \triangleleft N_{k}=G$ is a subnormal series. For $k \in\{1,2\}$ and $G$ finitely presented, semistability was proved by Mihalik $[17 ; 19]$. Those proofs easily generalize to the finitely generated case. The result that $\mathrm{G}$ is one-ended can be concluded from results of Cohen [2] or Stallings [27]. A geometric proof of this fact was given by Lee and Raymond [14]. We may assume that the index $\left[G: N_{k-1}\right]=\infty$, as $G$ is semistable at $\infty$ if and only if any subgroup of finite index is semistable at $\infty$.

Let $\mathcal{H}=\left\{h_{1}, h_{2}, \ldots, h_{n}\right\}$ be a finite generating set for $H$. Now, $G$ has generating set $S \equiv\left\{h_{1}, h_{2}, \ldots, h_{n}, a_{1}, a_{2}, \ldots, a_{m}, k_{1}, k_{2}, \ldots, k_{t}\right\}$ where, under the projection map $\rho: G \rightarrow G / N_{k-1}, \rho\left(k_{1}\right), \ldots, \rho\left(k_{t}\right)$ generate $G / N_{k-1}$ and the set $\left\{h_{1}, \ldots, h_{n}, a_{1}, \ldots\right.$, $\left.a_{m}\right\}$ is a subset of $N_{k-1}$. Let $\mathcal{K}=\left\{k_{1}, \ldots, k_{t}\right\}$. We also assume that conjugates of the $h_{i}$ by the $k_{j}$ are among $a_{1}, \ldots, a_{m}$ with the corresponding defining relations, say 
$k_{i} h_{j} k_{i}^{-1} \equiv a_{i j}$, and $k_{i}^{-1} h_{j} k_{i} \equiv b_{i j}$ for $i=1,2, \ldots, t$ and $j=1,2, \ldots, n$ so that $a_{i j}, b_{i j} \in\left\{a_{1}, a_{2}, \ldots, a_{m}\right\}$. Define $Q$ to be this set of conjugation relations,

$$
Q=\left\{k_{i} h_{j} k_{i}^{-1} a_{i j}^{-1}, k_{i}^{-1} h_{j} k_{i} b_{i j}^{-1}: i=1, \ldots, t \text { and } j=1, \ldots, n\right\} .
$$

Let $A$ be the subgroup of $N_{k-1}$ generated by $\mathcal{A}=\left\{h_{1}, \ldots, h_{n}, a_{1}, \ldots, a_{m}\right\}$. Let $A_{i}=N_{i} \cap A$ for $i \in\{1, \ldots, n-2\}$. Then the subnormal sequence

$$
H=A_{0} \triangleleft A_{1} \triangleleft \cdots \triangleleft A_{k-2} \triangleleft A
$$

has length $k-1$. The proof splits naturally into the two cases of whether or not $H$ has finite index in $A$. In the case where $H$ has finite index in $A$, we give a straightforward argument showing that $H$ is commensurated in $G$ and by our main theorem $G$ is semistable at $\infty$. Note that if $k=1$, this is the only case (since $A \subset N_{0}=H$ ). So when the proof of the first case is concluded, we are in position to apply an induction argument (with base case in hand) to the remaining case.

Suppose $H$ has finite index in $A$. Each point of $\Gamma(A, \mathcal{A})$ is within a bounded distance of $a H$ for any $a \in A$. In particular the Hausdorff distance between $H$ and $a H$ is bounded.

If $k \in \mathcal{K}^{ \pm 1}$ and $z \in k H$, then $z=k h$ for some $h \in H$. Note that $k h k^{-1} \in A$ (it is a product of the $a_{i j}^{ \pm 1}$ or $b_{i j}^{ \pm 1}$ ). Since $H$ has finite index in $A$, this point is close to $H$. As each point of $k H$ is close to $H$, left multiplying by $k^{-1}$ shows that each point of $H$ is close to $k^{-1} H$ for all $k \in \mathcal{K}^{ \pm 1}$. We have that $H$ is commensurated in $G$. The conditions of our main theorem are satisfied and so in the case $H$ has finite index in $A, G$ is semistable at $\infty$.

Now suppose $H$ has infinite index in $A$. The subnormal sequence $H=N_{0} \triangleleft N_{1} \triangleleft$ $\cdots \triangleleft N_{k-1} \triangleleft G$ has length $k$. Case 1 (or Mihalik's theorem [17]) shows that if $k=1$, then $G$ is semistable at $\infty$. Inductively, we assume that if $G^{\prime}$ is finitely generated and has a subnormal sequence of $H^{\prime}=N_{0}^{\prime} \triangleleft N_{1}^{\prime} \triangleleft \cdots \triangleleft N_{k-2}^{\prime} \triangleleft G^{\prime}$ of length $k-1$ such that $H^{\prime}$ is finitely generated and has infinite index in $G^{\prime}$, then $G^{\prime}$ is semistable at $\infty$.

In our case, $H$ has infinite index in $A$, and the $k-1$ length subnormal series $H=$ $A_{0} \triangleleft A_{1} \triangleleft \cdots \triangleleft A_{k-2} \triangleleft A$ implies that $A$ is semistable at $\infty$. Hence we may choose a finite set of $\mathcal{A}$-relations $P$ so that $\Gamma_{(A, \mathcal{A})}(P)$ is semistable. By using Lemma 3.7 or Remark 3.8, we may assume that if $r$ and $s$ are $\mathcal{A}$-rays at $v$ in $\Gamma_{(A, \mathcal{A})}(P)$, then $r$ and $s$ are properly homotopic $\operatorname{rel}\{v\}$ in $\Gamma_{(A, \mathcal{A})}(P)$. In this case, denote $\Gamma_{(G, S)}(P \cup Q)$ by $\widetilde{\Gamma}$ (where $Q$ is the set of conjugation relations defined at the beginning of this proof). If $v \in G$ (so $v$ is a vertex of $\widetilde{\Gamma}$ ) and $C_{v}$ is a compact subcomplex of $v \Gamma_{(A, \mathcal{A})}(P) \subset \widetilde{\Gamma}$, there is a compact subcomplex $D_{v}$ of $v \Gamma_{(A, \mathcal{A})}(P)$ such that if $r$ and $s$ are edge 
path rays at $w \in v \Gamma_{(A, \mathcal{A})}(P)-D_{v}$, then $r$ and $s$ are properly homotopic rel\{ $\left.v\right\}$ by a proper homotopy whose image does not intersect $C_{v}$. Hence, if $C$ is a compact subcomplex of $\widetilde{\Gamma}$ and we let $C_{v}=C \cap v \Gamma_{(A, \mathcal{A})}(P)$ (for the finite set of vertices $v$ such $\left.C \cap v \Gamma_{(A, \mathcal{A})}(P) \neq \varnothing\right)$ and let $D=\cup D_{v}$, then any two $\mathcal{A}$-rays $r$ and $s$ at $w \in v \Gamma_{(A, \mathcal{A})}(P)-D$ are properly homotopic $\operatorname{rel}\{w\}$ in $\widetilde{\Gamma}-C$.

We use $\mathcal{H}$-rays $r_{v}$, as defined in Lemma 4.5.

Choose a sequence of compact subcomplexes $\left\{C_{i}\right\}_{i=1}^{\infty}$ of $\widetilde{\Gamma}$ satisfying the following conditions:

$$
\bigcup_{i=1}^{\infty} C_{i}=\widetilde{\Gamma}
$$

(2) $\operatorname{St}\left(C_{i}\right)$ is contained in the interior of $C_{i+1}$, and the finite set of vertices $v$ such that $r_{v}$ intersects $C_{i}$ is a subset of $C_{i+1}$.

(3) If $r$ and $s$ are $\mathcal{A}$-rays both based at a vertex $v$ with images missing $C_{i}$, then $r$ and $s$ are properly homotopic $\operatorname{rel}\{v\}$ by a proper homotopy missing $C_{i-1}$.

For convenience define $C_{i}=\varnothing$ for $i<1$ and observe that conditions (1), (2), and (3) remain valid for all $C_{i}$. The next lemma concludes the proof of the second case and the theorem.

Lemma 5.2 If $v$ is a vertex of $\widetilde{\Gamma}$, and $s=\left(s_{1}, s_{2}, \ldots\right)$ is an $S$-ray at $v$, then $s$ is properly homotopic to $r_{v} \operatorname{rel}\{v\}$.

Proof Assume that $s$ has consecutive vertices $v=v_{0}, v_{1}, \ldots$ By construction, if $v_{j} \in C_{i}-C_{i-1}$, then $r_{v_{j}}$ avoids $C_{i-1}$. Assume $j$ is the largest integer such that $C_{j}$ avoids $s_{i}$. We will show $r_{v_{i-1}}$ is properly homotopic to $s_{i} * r_{v_{i}} \operatorname{rel}\left\{v_{i-1}\right\}$ by a proper homotopy $H_{i}$ with image avoiding $C_{j-2}$.

If $s_{i} \in \mathcal{A}^{ \pm 1}$, this is clear by condition (3) with $H_{i}$ avoiding $C_{j-1}$. If $s_{i} \in \mathcal{K}^{ \pm 1}$, then $s_{i} * r_{v_{i}}$ is properly homotopic $\operatorname{rel}\left\{v_{i-1}\right\}$ to an $\mathcal{A}$-ray, $t_{v_{i-1}}$ (using only 2-cells arising from $Q)$, and this homotopy has image in $\operatorname{St}\left(\operatorname{Im}\left(s_{i} * r_{v_{i}}\right)\right) \subset \widetilde{\Gamma}-C_{j-1}$. Since $t_{v_{i-1}}$ and $r_{v_{i-1}}$ are $\mathcal{A}$-rays with images avoiding $C_{j-1}$, condition (3) on the sets $C_{i}$ gives a proper homotopy between them $\operatorname{rel}\left\{v_{i-1}\right\}$, whose image avoids $C_{j-2}$. Patch these two proper homotopies together to obtain $H_{i}$.

Let $H$ be the homotopy $\operatorname{rel}\{v\}$ of $s$ to $r_{v}$, obtained by patching together the homotopies $H_{i}$. We need to check that $H$ is proper. Let $C \subset \widetilde{\Gamma}$ be compact. Choose an index $j$ such that $C \subseteq C_{j}$. Since $s$ is a proper edge path to infinity, choose an index $N$ such that all edges after the $N^{\text {th }}$ edge of $s$ avoid $C_{j+2}$. Then for all $i>N, H_{i}$ avoids $C_{j}$, so $H$ is proper.

This concludes the proof of the theorem. 


\section{Simple connectivity at $\infty$}

Recall, a connected locally finite CW-complex $X$ is simply connected at $\infty$ if for each compact set $C$ in $X$ there is a compact set $D$ in $X$ such that loops in $X-D$ are homotopically trivial in $X-C$. A group $G$ is simply connected at $\infty$ if given some (equivalently any; see Lee and Raymond [14, Theorem 3]) finite complex $X$ with $\pi_{1}(X)=G$, the universal cover of $X$ is simply connected at $\infty$.

If $G$ is a group and $H$ a subgroup of $G$ there are various notions for the number of ends of the pair $(G, H)$. Geoghegan [7, Chapter 14] gives a good account of these notions. In particular, the idea of the number of filtered ends of the pair $(G, H)$ is developed and compared to the standard number of ends of a pair. In any case, the number of filtered ends of the pair $(G, H)$ is greater than or equal to the number of standard ends of the pair. [7, Proposition 14.5.9] shows that if $H$ is a normal subgroup of $G$, then the number of ends of $G / H$, the standard number of ends of $(G, H)$ and the number of filtered ends of $(G, H)$ are all the same. Conner and Mihalik [3] show that if $G$ is a group with finite generating set $S$ and $Q$ is a finitely generated commensurated subgroup of $G$, then the number of filtered ends of $(G, Q)$ equals the number of ends of $\Lambda(S, Q, G)$.

Theorem 6.1 Suppose $G$ is a finitely presented group with finite generating set $S$ and $Q$ is a finitely presented, infinite commensurated subgroup of infinite index in $G$. If $Q$ or $\Lambda(S, Q, G)$ is one-ended, then $G$ is simply connected at $\infty$.

Proof Suppose $\mathcal{P}=\left\langle q_{1}, \ldots, q_{a}, k_{1}, \ldots, k_{b}: R\right\rangle$ is a finite presentation of the group $G$ such that the $q_{i}$ generate the infinite commensurated subgroup $Q$, no $k_{i}$ is an element of $Q$, and $R$ contains relations $R^{\prime}$ such that $\left\langle q_{1}, \ldots, q_{a}: R^{\prime}\right\rangle$ is a finite presentation of $Q$. Assume that $Q$ has infinite index in $G$. Let $X$ be the Cayley 2-complex of $\mathcal{P}$, $\tilde{X}$ the universal cover for $X$ and $\tilde{X}(Q, v) \subset \tilde{X}$ the copy of the universal cover of the Cayley 2-complex for $\left\langle q_{1}, \ldots, q_{a}: R^{\prime}\right\rangle$ containing $v$. Let $\mathcal{K}=\left\{k_{1}, \ldots, k_{b}\right\}$ and $\mathcal{Q}=\left\{q_{1}, \ldots q_{a}\right\}$.

Let $N_{1}$ be an integer such that if cosets $g Q$ and $h Q$ of $G$ are connected by an edge in $\tilde{X}$, then the Hausdorff distance between $g Q$ and $h Q$ in $\tilde{X}$ is at most $N_{1}$. For each relator $r \in R$, let $r^{\prime}$ be the word obtained from $r$ by removing $\mathcal{Q}$ letters. For each such (non-trivial) $r^{\prime}$ and edge loop in $\Lambda(S, Q, G)$ with edge label $r^{\prime}$, attach a 2-cell and call the resulting locally finite 2 -complex $\widehat{\Lambda}(S, Q, G)$. Note that $\Gamma(S, G)$ is the $1-$ skeleton of $\tilde{X}$. Extend the map $\rho: \Gamma(S, G) \rightarrow \Lambda(S, Q, G)$ (see Proposition 2.2) to $\rho: \tilde{X} \rightarrow \widehat{\Lambda}(S, Q, G)$. Let $C$ be a finite subcomplex of $\tilde{X}$. Let $d_{1} \geq 1$ be an integer such that for each vertex $v$ of $\rho(C)$, there is a $\mathcal{K}$-edge path in $\widehat{\Lambda}(S, Q, G)$ of length 
at most $d_{1}$ from $v$ to a vertex of $\widehat{\Lambda}(S, Q, G)-\rho(C)$. In particular, for each vertex $v$ of $\tilde{X}$, there is an edge path at $v$ of length at most $N_{1} d_{1}$ and with end point $w$ such that $\tilde{X}(Q, w) \cap C=\varnothing$. For each $k \in\left\{k_{1}, \ldots, k_{b}\right\}$ assume that $Q$ and $k Q$ are within Hausdorff distance $N_{1}$. Choose $N_{2}$ so that if $q_{1}$ and $q_{2}$ are two $Q$-vertices of $\tilde{X}$ with the edge path distance in $\tilde{X}$ between $q_{1}$ and $q_{2}$ less than or equal to $2 N_{1}+1$, then the edge path distance between $q_{1}$ and $q_{2}$ in $\widetilde{X}\left(Q, q_{1}\right)$ is at most $N_{2}$. In particular, there is a $\mathcal{Q}$-edge path between $q_{1}$ and $q_{2}$ of length at most $N_{2}$. Choose $N_{3}$ such that if $\alpha$ is an edge path loop at $* \in \tilde{X}$ of length at most $2 N_{1}+N_{2}+1$, then $\alpha$ is homotopically trivial in $\mathrm{St}^{N_{3}}(*)$.

Lemma 6.2 Suppose $G$ is a finitely presented group, $Q$ is a finitely presented, infinite commensurated subgroup of infinite index in $G, \mathcal{P}$ is a presentation of $G$ as above, and $X$ is the Cayley 2-complex of $\mathcal{P}$. If $\alpha$ is a $\mathcal{Q}-$ loop in $\tilde{X}$, with image in $\tilde{X}-$ $S t^{d_{1} N_{1} N_{3}}(C)$, then $\alpha$ is homotopically trivial in $\tilde{X}-C$.

Proof We may assume $*$ is the initial vertex of $\alpha$. If $\tilde{X}(Q, v) \cap C=\varnothing$, then as $\alpha$ is homotopically trivial in $\tilde{X}(Q, v)$, we are finished. If $\tilde{X}(Q, v) \cap C \neq \varnothing$, there is an edge path $\beta=\left(b_{1}, \ldots, b_{k}\right)$ at $v$ with $k \leq N_{1} d_{1}$ and with end point $w$ such that $\tilde{X}(Q, w) \cap C=\varnothing$. Let $v \equiv v_{0}, \ldots, v_{k} \equiv w$ be the consecutive vertices of $\alpha$. For each vertex $x$ of $\alpha$, there is an edge path of length at most $N_{1}$ from $x$ to a vertex of $\tilde{X}\left(Q, v_{1}\right)$ (if $b_{1}$ is a $\mathcal{Q}$-edge, this path is trivial) and hence $\alpha$ is homotopic rel $\{0,1\}$ to a loop $\left(b_{1}, \alpha_{1}, b_{1}^{-1}\right)$, where $\alpha_{1}$ is a $\mathcal{Q}$-loop in $\tilde{X}\left(Q, b_{1}\right)$, by a homotopy in $S t^{N_{3}}(\operatorname{Im}(\alpha))$. Inductively, $\alpha$ is freely homotopic to a $\mathcal{Q}$-loop $\alpha_{k}$ at the end point of $\beta$, by a homotopy in $S t^{k N_{3}}(\operatorname{Im}(\alpha)) \subset \tilde{X}-C$. As $\tilde{X}(Q, w) \cap C=\varnothing$ and $\operatorname{Im}\left(\alpha_{k}\right) \subset \tilde{X}(Q, w), \alpha_{k}$ (and hence $\alpha$ ) is homotopically trivial in $\tilde{X}-C$.

Case $1 Q$ is one-ended There are finitely many vertices $w_{1}, \ldots, w_{n} \in \tilde{X}$ such that $\tilde{X}\left(Q, w_{i}\right) \cap S t^{\left(d_{1} N_{1}+1\right) N_{3}}(C) \neq \varnothing$. As $\tilde{X}\left(Q, w_{i}\right)$ is one-ended, there is a compact subcomplex $D$ of $\tilde{X}$ such that $S t^{\left(d_{1} N_{1}+1\right) N_{3}}(C) \subset D$ and for all $i \in\{1, \ldots, n\}$ and vertices

$$
x, y \in \tilde{X}\left(Q, w_{i}\right)-D,
$$

$x$ and $y$ can be joined by a $\mathcal{Q}$-edge path in $\tilde{X}\left(Q, v_{i}\right)-S t^{\left(d_{1} N_{1}+1\right) N_{3}}(C)$. Now, suppose $\alpha$ is an arbitrary loop in $\tilde{X}-D$ with initial vertex $v$. Choose $L$ a positive integer such that if $q_{1}$ and $q_{2}$ are vertices of $\tilde{X}(Q, *)$ that are of distance $\leq N_{1}|\alpha|$ apart in $\tilde{X}$, then they are of distance $\leq L$ in $\tilde{X}(Q, *)$. Choose $E$ such that any edge path loop $\tau$ at a vertex $x$ of $\tilde{X}$, of length $\leq N_{1}|\alpha|+L$, is homotopically trivial in $S t^{E}(x)$. Let $\beta_{1}$ be a $\mathcal{Q}$-path in $\tilde{X}(Q, v)-S t^{\left(d_{1} N_{1}+1\right) N_{3}}(C)$ from $v$ to a point

$$
w \in \tilde{X}-\left(S t^{E}(C) \cup S t^{d_{1} N_{1} N_{3}+L}(C) \cup S t^{N_{1}|\alpha|}(D)\right) .
$$


Write the edge path $\alpha$ as $\left(e_{1}, \ldots, e_{m}\right)$ with consecutive vertices $v=v_{0}, \ldots, v_{m}$. As $w \in \tilde{X}(Q, v)$, there is an edge path $\tau_{1}$ of length at most $N_{1}$ from $w=w_{1}$ to $w_{2} \in \tilde{X}\left(Q, v_{2}\right)$. Let $\tau_{2}$ be an edge path of length at most $N_{1}$ from $w_{2}$ to $w_{3} \in$ $\tilde{X}\left(Q, v_{3}\right)$. Inductively, $\tau_{m}$ is an edge path of length at most $N_{1}$ from $w_{m}$ to a vertex $w_{m+1} \in \tilde{X}(Q, v)$. (Note that $\tau_{i}$ may be taken as the trivial path if $e_{i}$ is a $\mathcal{Q}$-edge.) As the edge path $\left(\tau_{1}, \ldots, \tau_{m}\right)$ has length at most $N_{1}|\alpha|$, there is a $\mathcal{Q}$-path $\lambda$ from $w_{m+1}$ to $w$ of length at most $L$. By the definition of $E$, the loop $\tau \equiv\left(\tau_{1}, \ldots, \tau_{m}, \lambda\right)$ at $w$ is homotopically trivial in $\tilde{X}-C$. Hence, it suffices to show that $\alpha$ is freely homotopic to $\tau$ in $\tilde{X}-C$. (See Figure 1.)

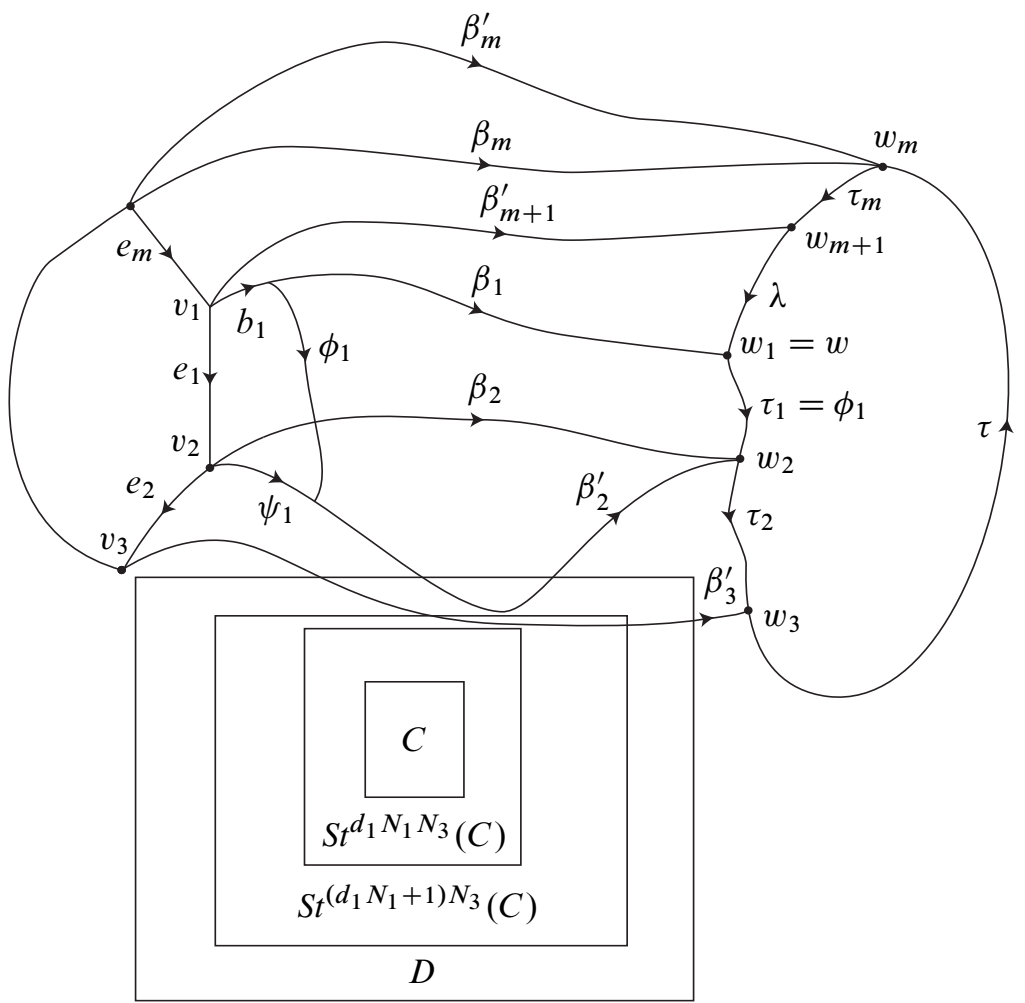

Figure 1

First note that each vertex of $\left(\tau_{1}, \ldots, \tau_{m}\right)$ is in $\tilde{X}-D$, since the vertex $w \in \tilde{X}-$ $S t^{N_{1}|\alpha|}(D)$. Next, write $\beta_{1}$ as the edge path $\left(b_{1}, \ldots, b_{s}\right)$. Let $\phi_{0}=e_{1}$ and let $\phi_{i}$ be an edge path of length at most $N_{1}$ from the end point of $b_{i}$ to a point of $\tilde{X}\left(Q, v_{2}\right)$. Let $\psi_{i}$ be a $\mathcal{Q}$-edge path of length at most $N_{2}$ from the end point of $\phi_{i-1}$ to the end point of $\phi_{i}$. (Choose $\phi_{s}=\tau_{1}$.) Then the loop $\left(\phi_{i-1}, \psi_{i}, \phi_{i}^{-1}, b_{i}^{-1}\right)$ has length 
at most $2 N_{1}+N_{2}+1$ and is homotopically trivial by a homotopy in the $N_{3}$-star of the initial point of $b_{i}$. String together these homotopies, and we have that the edge path $\left\langle e_{1}^{-1}, \beta_{1}, \tau_{1}\right)$ is homotopic $\operatorname{rel}\{0,1\}$ to the $\mathcal{Q}$-edge path $\beta_{2}^{\prime} \equiv\left(\psi_{1}, \ldots, \psi_{m}\right)$ by a homotopy with image in

$$
S t^{N_{3}}\left(\operatorname{Im}\left(\beta_{1}\right)\right) \subset \tilde{X}-S t^{d_{1} N_{1} N_{3}}(C) .
$$

By the definition of $D$, there is an $\mathcal{Q}$-edge path $\beta_{2}$ with the same end points as $\beta_{2}^{\prime}$ and with image in $\tilde{X}-S t^{\left(d_{1} N_{1}+1\right) N_{3}}(C)$. By Lemma 6.2, $\beta_{2}$ and $\beta_{2}^{\prime}$ are homotopic $\operatorname{rel}\{0,1\}$ by a homotopy in $\tilde{X}-C$. Continue inductively until $\beta_{m}$ and $\beta_{m+1}^{\prime}$ are defined. Since

$$
w \in \tilde{X}-S t^{d_{1} N_{1} N_{3}+L}(C),
$$

the path $\lambda$ (of length at most $L$ ) has image in $\tilde{X}-S t^{d_{1} N_{1} N_{3}}(C)$. By Lemma 6.2, the $\mathcal{Q}$-loop $\left(\beta_{m+1}^{\prime}, \lambda, \beta_{1}^{-1}\right)$ is homotopically trivial in $\tilde{X}-C$.

Case $2 \boldsymbol{\Lambda}(\boldsymbol{S}, \boldsymbol{Q}, \boldsymbol{G})$ is one-ended The letters $N_{1}, N_{2}$ and $N_{3}$ remain as in Case 1 and we recycle letters used for any other constant.

Given a finite subcomplex $C$ of $\tilde{X}$, consider

$$
\rho(C) \subset \widehat{\Lambda}(S, Q, G) .
$$

Choose $D$ a finite subgraph of $\Lambda(S, Q, G)$ such that any two vertices of $\widehat{\Lambda}(S, Q, G)-$ $D$ can be connected by a path in $\hat{\Lambda}(S, Q, G)-\rho\left(S t^{N_{3}}(C)\right)$. For each vertex $v$ of $D$ choose a path $\bar{\alpha}_{v}$ from $v$ to a vertex of $\widehat{\Lambda}(S, Q, G)-D$. If $v$ is a vertex of $\widehat{\Lambda}(S, Q, G)-D$, let $\bar{\alpha}_{v}$ be the trivial path. Let $N$ be the length of the longest path $\bar{\alpha}_{v}$ for $v \in D$. If $v$ is a vertex of $\tilde{X}$ such that $\rho(v) \in D$, let $\alpha_{v}$ be an edge path of the form $\left(\beta_{1}, \ldots, \beta_{m}\right)$ where each $\beta_{i}$ has length at most $N_{1}$, and $\rho\left(\beta_{i}\right)$ has the same end points as the $i^{\text {th }}$ edge of $\bar{\alpha}_{\rho(v)}$ (so $\left|\alpha_{v}\right| \leq N_{1} N$ ). In analogy with previous terminology, we call $\alpha_{v}$ an $N_{1}$-approximate lift of $\bar{\alpha}_{\rho(v)}$. If $\rho(v) \notin D$, let $\alpha_{v}$ be the trivial path.

Choose an integer $M$ such that if $v$ and $w$ are adjacent vertices of $\operatorname{St}(D)$, then there is an edge path $\bar{\alpha}_{v, w}$ in $\widehat{\Lambda}(S, Q, G)-\rho\left(S t S t^{N_{3}}(C)\right)$ of length at most $M$ from the end point of $\bar{\alpha}_{v}$ to the end point of $\bar{\alpha}_{w}$. Choose an integer $B$ such that if $\beta$ is a $\tilde{X}$-edge path of length at most $(2 N+M) N_{1}+1$ connecting $*$ (the vertex of $\tilde{X}$ corresponding to the identity element of $G$ ) to a vertex $q \in Q$, then there is a $\mathcal{Q}$-edge path of length at most $B$ connecting $*$ to $q$. Choose an integer $A$ such that if $\beta$ is an edge path loop at $*$ of length at most $(2 N+M) N_{1}+B+1$, then $\beta$ is homotopically trivial in $S t^{A}(*)$.

We next show that if $\beta$ is an edge path loop in $\tilde{X}-S t^{A}(C)$, then $\beta$ is freely homotopic to a loop $\hat{\beta}$ by a homotopy in $\widetilde{X}-C$, where $\hat{\beta}$ can be chosen so that for each vertex $v$ 
of $\hat{\beta}, \rho(v) \notin \rho\left(S t S t^{N_{3}}(C)\right)$. If $e$ is a directed edge of $\tilde{X}$ or $\Lambda(S, Q, G)$, with initial point $a$ and terminal point $b$, then let $[a, b]$ represent this edge. Suppose $\beta$ is the edge path $\left(d_{1}, d_{2}, \ldots, d_{n}\right)$ with consecutive vertices $b_{1}, \ldots, b_{n+1}$. If (cyclically) neither $\rho\left(b_{i}\right)$ nor $\rho\left(b_{i+1}\right)$ is in $D$, then let $\widehat{\beta}_{i}$ be the single edge $d_{i}$. Otherwise, $\rho\left(b_{i}\right)$ and $\rho\left(b_{i+1}\right)$ belong to $\operatorname{St}(D)$. In this case, consider the edge path $\delta_{i} \equiv\left(\alpha_{b_{i}}^{-1}, d_{i}, \alpha_{b_{i+1}}\right)$ of $\tilde{X}$.

If $\rho\left(b_{i}\right) \neq \rho\left(b_{i+1}\right)$, the edge path $\bar{\alpha}_{\rho\left(b_{i}\right), \rho\left(b_{i+1}\right)}$ joins the end points of $\rho\left(\delta_{i}\right)$ and has length at most $M$. Let $\alpha_{i}$ be an $N_{1}$-approximate lift of $\bar{\alpha}_{\rho\left(b_{i}\right), \rho\left(b_{i+1}\right)}$ to the initial point of $\delta_{i}$ (otherwise, let $\alpha_{i}$ be the trivial path at the initial point of $\delta_{i}$ ).

Note that the end point of $\alpha_{i}$ and the end point of $\delta_{i}$ belong to the same left $Q$-coset. As the length of $\left(\alpha_{i}^{-1}, \delta_{i}\right)$ is at most $(2 N+M) N_{1}+1$, there is a $\mathcal{Q}$-edge path $\gamma_{i}$ of length at most $B$ from the initial point to the end point of $\left(\alpha_{i}^{-1}, \delta_{i}\right)$. The loop $\left(\gamma_{i}^{-1}, \alpha_{i}^{-1}, \delta_{i}\right)$ has length $\leq 2 N+M+B+1$ and so is homotopically trivial in $\tilde{X}-C$ (by the definition of $A$ ). Let $\hat{\beta}_{i}=\left(\alpha_{i}, \gamma_{i}\right)$, for $i \in\{1, \ldots, n\}$. Let $\hat{\beta}$ be the loop $\left(\hat{\beta}_{1}, \ldots, \hat{\beta}_{n}\right)$. Combining homotopies shows that $\beta$ is freely homotopic to $\hat{\beta}$ by a homotopy in $\tilde{X}-C$. As $\rho\left(\alpha_{i}\right)$ avoids $\rho\left(\operatorname{StSt}^{N_{3}}(C)\right), \rho(\hat{\beta})$ avoids $\rho\left(S t S t^{N_{3}}(C)\right)$. (See Figure 2.)

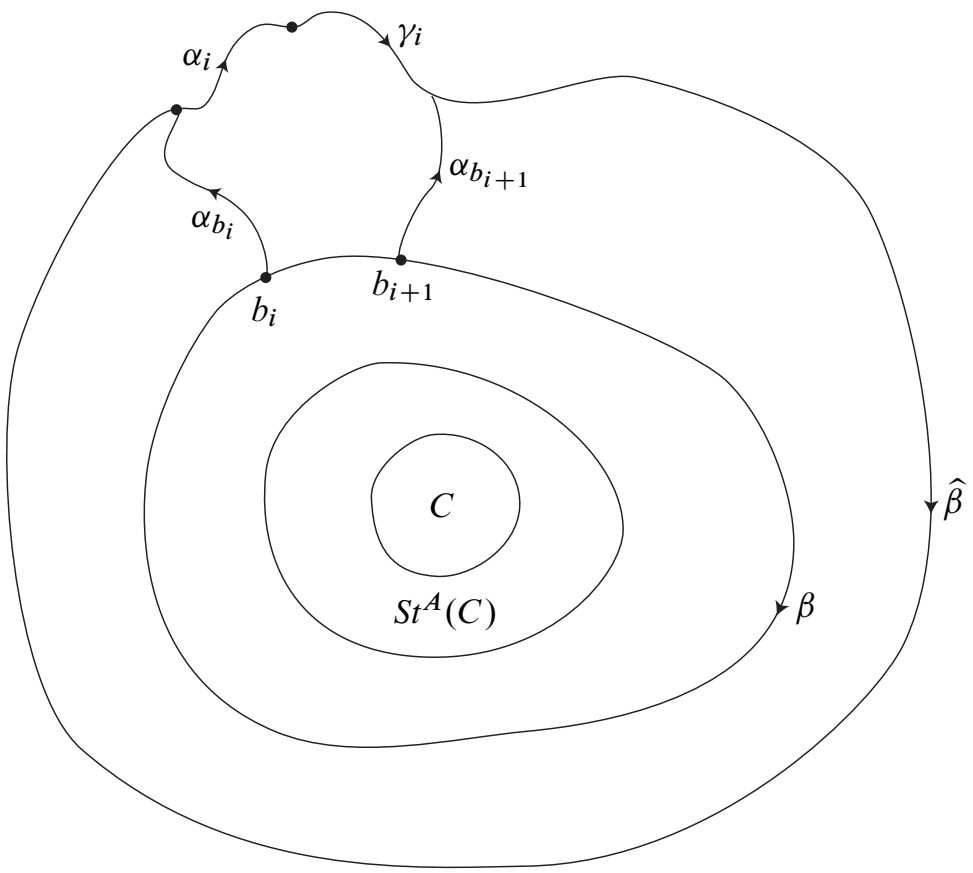

Figure 2 
We conclude the proof of Case 2 by showing $\hat{\beta}$ is homotopically trivial in $\tilde{X}-C$. The proof is analogous to the closing argument of Case 1. Let $v$ be the initial vertex of $\hat{\beta}$. Choose $L$ a positive integer such that if $q_{1}$ and $q_{2}$ are vertices of $\tilde{X}(Q, *)$ that are of distance at most $N_{1}|\hat{\beta}|$ apart in $\tilde{X}$, then they are of distance at most $L$ in $\tilde{X}(Q, *)$. Choose $E$ such that any edge path loop $\tau$ at a vertex $x$ of $\tilde{X}$ and of length at most $N_{1}|\hat{\beta}|+L$ is homotopically trivial in $S t^{E}(x)$. Let $\beta_{1}$ be a $\mathcal{Q}$-path from $v$ to a point $w \in \widetilde{X}-S t^{E}(C)$. Write the edge path $\hat{\beta}$ as $\left(e_{1}, \ldots, e_{m}\right)$ with consecutive vertices $v \equiv v_{1}, v_{2}, \ldots, v_{m}$. As $w \in \tilde{X}(Q, v)$ there is an edge path $\tau_{1}$ of length at most $N_{1}$ from $w$ to $w_{2} \in \tilde{X}\left(Q, v_{2}\right)$. Let $\tau_{2}$ be an edge path of length at most $N_{1}$ from $w_{2}$ to $w_{3} \in \tilde{X}\left(Q, v_{3}\right)$. Inductively, $\tau_{m}$ is an edge path of length at most $N_{1}$ from $w_{m}$ to a vertex $w_{m+1} \in \tilde{X}(Q, v)$. (Note that $\tau_{i}$ may be taken as the trivial path if $e_{i}$ is a $\mathcal{Q}$-edge.) As the edge path $\left(\tau_{1}, \ldots \tau_{m}\right)$ begins and ends in $\tilde{X}(Q, v)$ and has length at most $N_{1}|\hat{\beta}|$, there is a $\mathcal{Q}$-path $\lambda$ from $w_{m+1}$ to $w$ of length at most $L$. By the definition of $E$, the loop $\tau \equiv\left(\tau_{1}, \ldots, \tau_{m}, \lambda\right)$ at $w$ is homotopically trivial in $\tilde{X}-C$. Hence, it suffices to show that $\alpha$ is freely homotopic to $\tau$ in $\tilde{X}-C$.

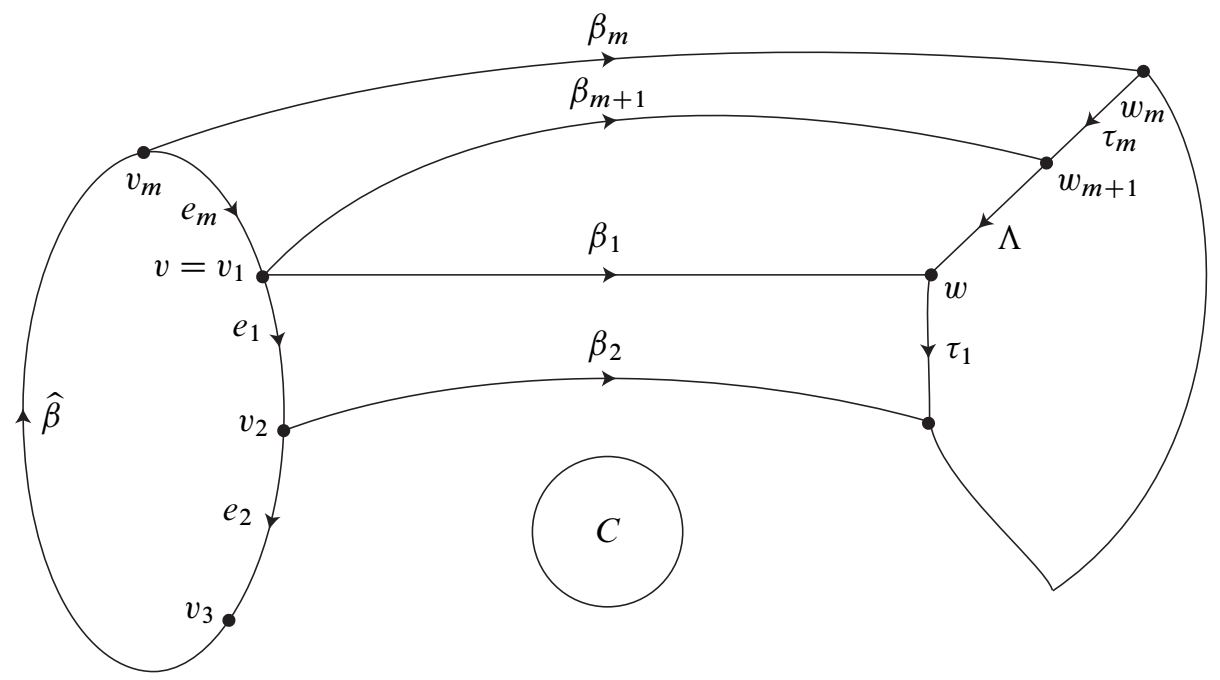

Figure 3

Each vertex $b$ of $\beta_{1}$ is such that $\rho(v)=\rho(b) \in \hat{\Lambda}(S, Q, G)-\rho\left(S t S t N_{3}(C)\right)$ and so the image of $\beta_{1}$ avoids $S t^{N_{3}}(C)$. As in Case 1, this implies that the path $\left(e_{1}^{-1}, \beta_{1}, \tau_{1}\right)$ is homotopic rel\{0,1\} to a $\mathcal{Q}$-edge path $\beta_{2}$ by a homotopy with image in

$$
S t^{N_{3}}\left(\operatorname{Im}\left(\beta_{1}\right)\right) \subset \tilde{X}-C .
$$

Each vertex $b$ of $\beta_{2}$ is such that $\rho(b)=\rho\left(v_{2}\right) \in \widehat{\Lambda}(S, Q, G)-\rho\left(S t S t^{N_{3}}(C)\right)$ and so the image of $\beta_{2}$ avoids $S t^{N_{3}}(C)$. The path $\left(e_{2}^{-1}, \beta_{2}, \tau_{2}\right)$ is homotopic rel\{0,1\} to 
a $\mathcal{Q}$-edge path $\beta_{3}$ by a homotopy with image in $S t^{N_{3}}\left(\operatorname{Im}\left(\beta_{2}\right)\right) \subset \tilde{X}-C$. Continue inductively until $\beta_{m+1}$ is defined (as a $\mathcal{Q}$-path from $v$ to $w_{m+1}$ ). As

$$
\rho(v) \in \hat{\Lambda}(S, Q, G)-\rho\left(S t S t^{N_{3}}(C)\right),
$$

the $\mathcal{Q}$-loop $\left(\beta_{1}, \lambda^{-1}, \beta_{m+1}^{-1}\right)$ has image in $\tilde{X}(Q, v) \subset \tilde{X}-C$, and so is homotopically trivial in $\tilde{X}-C$. (See Figure 3.) Combining homotopies produces a null homotopy of $\hat{\beta}$ with image in $\tilde{X}-C$.

\section{References}

[1] A Borel, Density and maximality of arithmetic subgroups, J. Reine Angew. Math. 224 (1966) 78-89 MR0205999

[2] D E Cohen, Groups of cohomological dimension one, Lecture Notes in Mathematics 245, Springer, New York (1972) MR0344359

[3] G Conner, M L Mihalik, Commensurated subgroups and ends of groups, J. Group Theory 16 (2013) 107-139 MR3008315

[4] M W Davis, Groups generated by reflections and aspherical manifolds not covered by Euclidean space, Ann. of Math. 117 (1983) 293-324 MR690848

[5] M J Dunwoody, The accessibility of finitely presented groups, Invent. Math. 81 (1985) 449-457 MR807066

[6] H Freudenthal, Über die Enden topologischer Räume und Gruppen, Math. Z. 33 (1931) 692-713 MR1545233

[7] R Geoghegan, The shape of a group - connections between shape theory and the homology of groups, from: "Geometric and algebraic topology", (H Toruńczyk, S Jackowski, S Spież, editors), Banach Center Publ. 18, PWN, Warsaw (1986) 271-280 MR925870

[8] R Geoghegan, Topological methods in group theory, Graduate Texts in Mathematics 243, Springer, New York (2008) MR2365352

[9] R Geoghegan, M L Mihalik, Free abelian cohomology of groups and ends of universal covers, J. Pure Appl. Algebra 36 (1985) 123-137 MR787167

[10] H Hopf, Enden offener Räume und unendliche diskontinuierliche Gruppen, Comment. Math. Helv. 16 (1944) 81-100 MR0010267

[11] B Jackson, End invariants of group extensions, Topology 21 (1982) 71-81 MR630881

[12] F E A Johnson, Manifolds of homotopy type $K(\pi, 1)$, $I$, Proc. Cambridge Philos. Soc. 70 (1971) 387-393 MR0290358

[13] F E A Johnson, On the first end invariant of an exact sequence, Mathematika 22 (1975) 60-70 MR0427483 
[14] R Lee, F Raymond, Manifolds covered by Euclidean space, Topology 14 (1975) 49-57 MR0365581

[15] V M Lew, The semistability at infinity for multiple extension groups, $\mathrm{PhD}$ thesis, Cornell University (1993) MR2689313

[16] G A Margulis, Discrete groups of motions of manifolds of nonpositive curvature, from: "Proceedings of the International Congress of Mathematicians, Vol. 2", Canad. Math. Congress, Montreal, Que. (1975) 21-34 MR0492072 In Russian; translated in Transl., Ser. II, Am. Math. Soc. 109 (1977), 33-45

[17] M L Mihalik, Semistability at the end of a group extension, Trans. Amer. Math. Soc. 277 (1983) 307-321 MR690054

[18] M L Mihalik, Ends of groups with the integers as quotient, J. Pure Appl. Algebra 35 (1985) 305-320 MR777262

[19] M L Mihalik, Ends of double extension groups, Topology 25 (1986) 45-53 MR836723

[20] ML Mihalik, Semistability at $\infty$ of finitely generated groups, and solvable groups, Topology Appl. 24 (1986) 259-269 MR872498

[21] ML Mihalik, Semistability at $\infty, \infty$-ended groups and group cohomology, Trans. Amer. Math. Soc. 303 (1987) 479-485 MR902779

[22] M L Mihalik, Semistability of Artin and Coxeter groups, J. Pure Appl. Algebra 111 (1996) 205-211 MR1394352

[23] M L Mihalik, S T Tschantz, One relator groups are semistable at infinity, Topology 31 (1992) 801-804 MR1191381

[24] ML Mihalik, S T Tschantz, Semistability of amalgamated products and HNNextensions, Mem. Amer. Math. Soc. 471, Amer. Math. Soc. (1992) MR1110521

[25] J S Profio, Using subnormality to show the simple connectivity at infinity of a finitely presented group, Trans. Amer. Math. Soc. 320 (1990) 281-292 MR961627

[26] L C Siebenmann, The obstruction to finding a boundary for an open manifold, $\mathrm{PhD}$ thesis, Princeton University (1965)

[27] J Stallings, Group theory and three-dimensional manifolds, Yale Mathematical Monographs 4, Yale University Press, New Haven, Conn. (1971) MR0415622

[28] G A Swarup, On the cut point conjecture, Electron. Res. Announc. Amer. Math. Soc. 2 (1996) 98-100 MR1412948

Math Department, Brigham Young University

275 TMCB, Provo, UT 84602, USA

Mathematics Department, Vanderbilt University

Nashville, TN 37240, USA

conner@math.byu.edu, michael.1.mihalik@vanderbilt.edu

Received: 16 September $2013 \quad$ Revised: 3 March 2014 